\title{
Bulk magnetic properties of $\mathrm{CdFe}_{2} \mathrm{O}_{4}$ in nano-regime
}

\author{
RUCHA DESAI, R V MEHTA, R V UPADHYAY*, AMITA GUPTA ${ }^{*}$, A PRANEET ${ }^{\dagger}$ and \\ K V RAO \\ Department of Physics, Bhavnagar University, Bhavnagar 364 002, India \\ ${ }^{\dagger}$ Department of Metallurgical Engineering, Institute of Technology, Banaras Hindu University, Varanasi 221 005, India \\ \#Materials Science-Tmfy-MSE, Royal Institute of Technology, Stockholm, Sweden
}

MS received 6 December 2006; revised 19 April 2007

\begin{abstract}
Cadmium ferrite particles have been synthesized using co-precipitation technique followed by a low temperature $\left(600^{\circ} \mathrm{C}\right)$ annealing in a time scale much shorter than reported in literature. Incorporation of sodium chloride during annealing helps to form a single phase spinel structure with a final particle size of around $50 \mathrm{~nm}$. Even at such a short length scale we observe the overall magnetic properties to be similar to those of the bulk. The observed magnetic properties can be explained on the basis of an anti-ferromagnetic core with a shell containing 'ferromagnetic-like', but canted spin structure.
\end{abstract}

Keywords. Cadmium ferrite; fine particle; disordered magnetic state; cation distribution.

\section{Introduction}

In recent years, the design and synthesis of nano-magnetic particles have been the focus of intense fundamental and applied research with special emphasis on their unusual or/and enhanced properties (Herzer et al 2005). In particular, spinel ferrite shows interesting magnetic properties in its nano-crystalline form compared to those of micron size bulk particles. Zinc ferrite has a normal spinel structure with $\mathrm{Zn}^{2+}$ and $\mathrm{Fe}^{3+}$ ions occupying tetrahedral $\mathrm{A}$ and octahedral B-sites, respectively (Hastings and Corliss 1956). It exhibits anti-ferromagnetic ordering below $9 \mathrm{~K}$. On the other hand, ultra fine zinc ferrite particles of several nanometers in size have remarkably large magnetization than the bulk materials (Sato et al 1987, 1990, 1992). A neutron diffraction experiment on these nanomagnetic zinc ferrite particles indicates that with decreasing particle size, occupancy of $\mathrm{Fe}^{3+}$ ions at the tetrahedral A-site increases (Kamiyama et al 1992). According to Lotgering (1966), these A-site ions form magnetic clusters consisting of $\mathrm{Fe}^{3+}$ ions at the B-site through A-B interaction. The number of clusters increases with decreasing particle size which eventually leads to increase in magnetization. Similar changes in magnetic properties with size were also observed in ultra fine cadmium ferrite synthesized by co-precipitation and high energy ball milled (HEMB) techniques (Yokoyama et al 1996, 1998; Chinnasamy et al 2001; Mahmoud et al 2003; Elkins et al 2005).

In $\mathrm{CdFe}_{2} \mathrm{O}_{4}$, fine particles synthesized by chemical route have given the magnetization value as high as $89 \mathrm{emu} / \mathrm{g}$

*Author for correspondence (rvu@bhavuni.edu) at $6 \mathrm{~K}$. Yokoyama et al (1996) observed that the system exhibits ferromagnetism and nearly $54 \%$ of the $\mathrm{Fe}^{3+}$ ions occupy the A-site in contrast to $0 \%$ for the bulk materials with normal spinel structure. This enhanced occupancy of $\mathrm{Fe}^{3+}$ ions in $\mathrm{CdFe}_{2} \mathrm{O}_{4}$ compared to $\mathrm{ZnFe}_{2} \mathrm{O}_{4}$ (14\%) is explained by considering higher octahedral preferential energy of $\mathrm{Cd}^{2+}(-29.1 \mathrm{kcal} / \mathrm{mol})$ ions to that of $\mathrm{Zn}^{2+}$ ions $(-31.6 \mathrm{kcal} / \mathrm{mol})$. They have further observed that even the $\mathrm{CdFe}_{2} \mathrm{O}_{4}$ prepared by ceramic technique exhibits ferromagnetic ordering at room temperature (Yokoyama et al 1998). Two different types of spin structure were suggested to explain these observed properties, i.e. (i) a core shell spin structure with a ferromagnetic core and inactive magnetic shell and (ii) non-collinear spin arrangement over the particle volume. In contrast, Chinnasamy et al (2001), using high field Mössbauer spectroscopy technique, showed that the ultra fine cadmium ferrite, synthesized by HEBM technique, behaves as mixed spinel on reducing the particle size $(<60 \mathrm{~nm})$ and exhibits ferrimagnetic ordering with canting at both the A and B sites. However, Mahmoud et al (2003) have shown recently that in cadmium ferrite A-site spins are collinear and only B-site moments are canted. They also show that the evolution from an anti-ferromagnetic ordering to a complex magnetic state (characterized by a local canted structure) is due primarily to small inversion of $\mathrm{Fe}^{3+}$ magnetic ions in cadmium ferrite.

In all the above investigations the bulk cadmium ferrite samples were prepared using double sintering ceramic technique. In this technique stoichiometric mixture of pure iron oxides and cadmium oxides are mixed and calcined between 900 and $1000^{\circ} \mathrm{C}$ for a few hours. After this precalcination the powder is pelletized and sintered at $1000^{\circ} \mathrm{C}$ for more than $15 \mathrm{~h}$. In order to get fine particles, bulk 
sample is ball milled using high energy ball milling technique. The degree of inversion starting from the normal spinel increases with ball milling time. Furthermore, the whole procedure is time consuming and one cannot get rid of the contamination from the ball during HEBM.

In the present paper, we describe a method to synthesize cadmium ferrite nano particles in a much short time span of a few hours. The method used by us is unique because (i) single phase Cd-ferrite nanoparticles were synthesized below $700^{\circ} \mathrm{C}$ resulting in a high crystalline structure, (ii) the final particle size is below $60 \mathrm{~nm}$ (diameter) i.e. in nano regime and (iii) the magnetic properties of this currently obtained nano-Cd-ferrite are identical to the bulk.

Our method can be extended to synthesize ferrites in the nano-regime without compromising the magnetic properties. The results are achieved in a much shorter time scale that renders industrial applications quite attractive.

\section{Experimental}

The starting materials used were of AR grade $\mathrm{CdCl}_{2} \cdot \mathrm{H}_{2} \mathrm{O}$ and $\mathrm{FeCl}_{3} \cdot 6 \mathrm{H}_{2} \mathrm{O}$. The chlorides were mixed in proper proportion and dissolved in distilled water to get aqueous salt solutions. The precipitates were obtained using $\mathrm{NaOH}$ as a precipitant under constant stirring while maintaining the reaction at a $\mathrm{pH}$ value of $10 \cdot 5$. The precipitate was then digested at $80^{\circ} \mathrm{C}$ for 30 min maintaining the same $\mathrm{pH}$ value of $10 \cdot 5$. Aliquots of the reaction mixture was collected and washed with distilled water and finally followed by acetone wash.

From these precipitates, in order to obtain single phase $\mathrm{Cd}$-ferrite nanoparticles, we adopted a new approach. We mixed homogeneously $1: 1$ ratio of $\mathrm{NaCl}$ and the 'as obtained' precipitates, pelletized and annealed at $600^{\circ} \mathrm{C}$ for $4 \mathrm{~h}$, after which the pellet was crushed, and washed with distilled water so as to remove $\mathrm{NaCl}$ completely. The melting point of $\mathrm{NaCl}$ was $810^{\circ} \mathrm{C}$, therefore, in the present case, the $\mathrm{NaCl}$ was not melted, but the addition of this inhibits the process of agglomeration. Recently, similar procedure was adopted to convert as synthesized facecentred cubic FePt nanoparticles to monodispersed facecentred tetragonal FePt nanoparticles without agglomerating the particles (Elkins et al 2005). We illustrate these observations from XRD studies on three samples: (i) as prepared precipitate (sample A), (ii) the 'asobtained' precipitate powder pelletized and annealed at $600^{\circ} \mathrm{C}$ for $4 \mathrm{~h}$ in air and subsequently pulverized (sample $\mathrm{B}$ ), and (iii) sample $\mathrm{C}$ in which the precipitate powder has been mixed with $\mathrm{NaCl}$ in the annealing process described above in order to obtain single phase $\mathrm{Cd}$-ferrite particles.

X-ray diffraction measurements of the above three powder samples were performed using Philips X-pert Diffractometer with $\mathrm{CuK}_{\alpha}$ radiation and the generator was kept operating at $40 \mathrm{kV} / 30 \mathrm{~mA}$. X-ray powder diffraction data were obtained in a $2 \theta$ scanning range from $25-80^{\circ}$ with a step size of $0.02^{\circ}(2 \theta)$.
The refinement of the structure was carried out with the Rietveld Refinement Program-DBWS-9006PC, developed by Dr H Marciniat, Poland, for X-ray powder and neutron powder diffraction data. The structure model and initial structure parameters were taken as follows: space group $F d 3 m$; metal and iron atoms were in the Wyckoff $8 a$ and $16 d$ special positions and $\mathrm{O}$ atoms in the $32 e$ special position. Moreover, both octahedral and tetrahedral cation and oxygen sites are assumed to be fully occupied. Diffraction profiles were modeled by using a multi-term Simpson's rule integration of the pseudo-Voigt function. The quality of the data fit to the experimental data were checked by using the following parameters: the goodness of fit, $\chi^{2}$ that must tend to 1 and 2, reliability factors, $R_{\mathrm{p}}$ and $R_{\mathrm{wp}}$ (weighted differences between measured and calculated values) that must be close to or less than $10 \%$. The refined crystallographic parameters were: the scale factor, the lattice parameter, $a$, the oxygen positional parameter, $u$, the mean particles size and the degree of inversion, $x$.

Transmission electron microscopic images were recorded using Philips, Technai-20 instruments, Sophisticated Instrumentation Centre for Applied Research and Testing (SICART), Gujarat. Instrument specifications to capture the images are accelerating voltage fixed at $200 \mathrm{kV}$, objective lens point resolution, $0.27 \mathrm{~nm}$ or better and line resolution, $2.0 \mathrm{~nm}$ or better.

Infrared spectra were recorded on the powder samples in $\mathrm{KBr}$ matrix using Thermonicolate FTIR spectrophotometer model IR200 at $300 \mathrm{~K}$.

Room temperature magnetization measurement was carried out using EG\&G 155 VSM. The instrument was calibrated before hand with Ni standard. The temperature dependent magnetization and the magnetic hysteresis, $M(H)$ loop, was carried out using quantum design $\mathrm{MPMS}_{2}$ SQUID magnetometer. Frequency dependent real and imaginary parts of the a.c. susceptibility measurements were carried out from 77-300 K using a high precision custom made susceptometer (Ström 1998) with room temperature sensitivity matching that of a SQUID.

\section{Results and discussion}

X-ray diffraction patterns for as prepared sample (A) is shown in figure 1a. The pattern is very complex and it is composed of lines corresponding to cadmium ferrite, $\mathrm{Cd}(\mathrm{OH})_{2}, \mathrm{Fe}_{2} \mathrm{O}_{3}$ and $\mathrm{FeO}(\mathrm{OH})$. The major contribution to the phase is $\mathrm{Cd}(\mathrm{OH})_{2}$. Pure cadmium ferrite is formed partially and the peak is very broad. The sample annealed at $600^{\circ} \mathrm{C}$ without $\mathrm{NaCl}$ (sample B) shows admixture of two phases (figure 1b). One corresponds to spinel cadmium ferrite phase and other impurity phase at $2 \theta$ value, $32^{\circ}$, corresponds to $\alpha-\mathrm{Fe}_{2} \mathrm{O}_{3}$ phase. The particle diameter $\left(D_{\mathrm{XR}}\right)$ obtained from the Scherrer's formalism for most intense peak (311) comes out to be $43 \mathrm{~nm}$ and the lattice constant for $\mathrm{CdFe}_{2} \mathrm{O}_{4}$ phase is $0.8692 \mathrm{~nm}$, which agrees 
with the bulk sample $(0.8697 \mathrm{~nm})$. In contrast to the above, the sample heated with $\mathrm{NaCl}$ (sample C) exhibits well crystalline single phase structure at the same annealing temperature. This suggests that because of direct heating
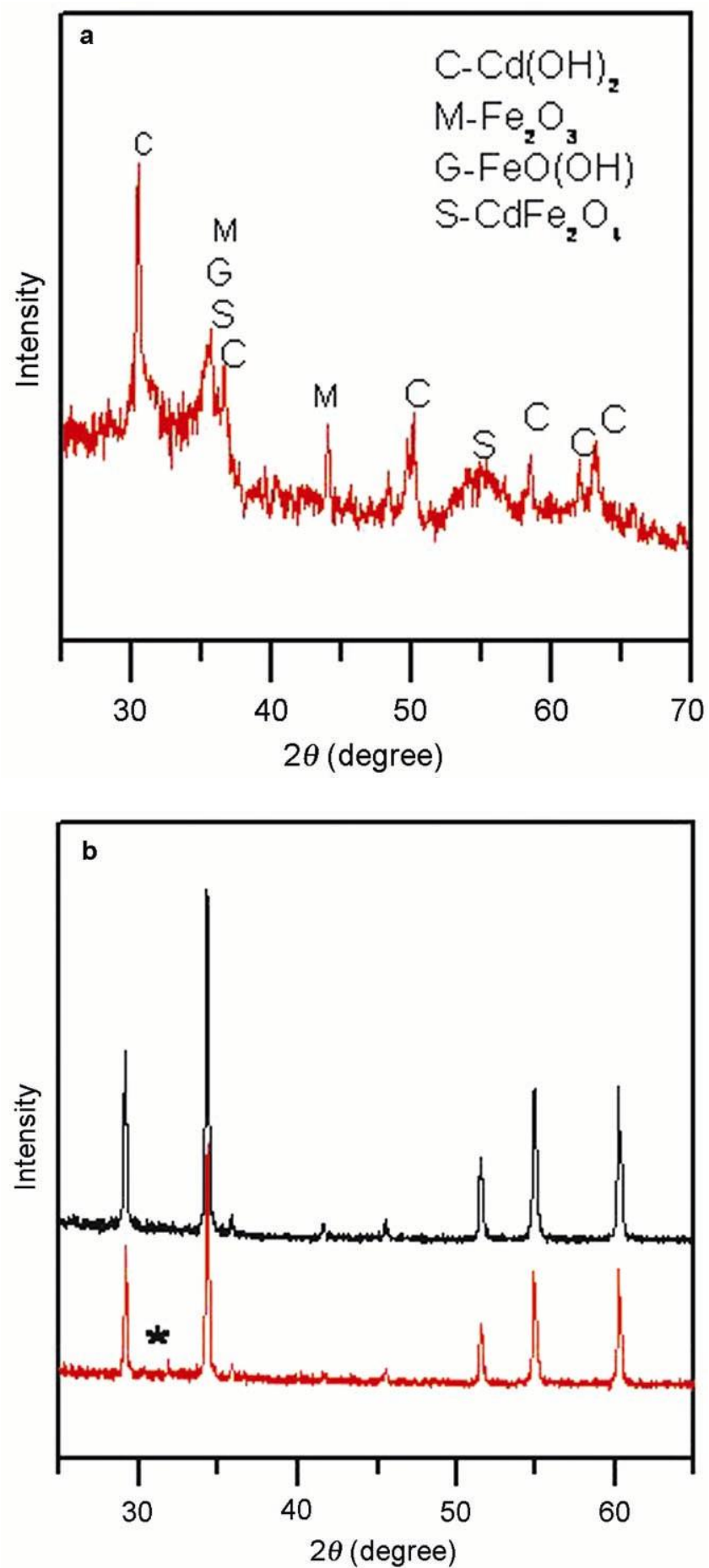

Figure 1. a. X-ray diffraction pattern for as prepared sample and $\mathbf{b}$. X-ray diffraction pattern for samples annealed at $600^{\circ} \mathrm{C}$ without $\mathrm{NaCl}$ (lower curve) and annealed at $600^{\circ} \mathrm{C}$ with $\mathrm{NaCl}$ (upper curve) as described in the text. The ${ }^{*}$ indicates the presence of impurity phase of precursor the formation of $\mathrm{Fe}_{2} \mathrm{O}_{3}$ phase takes place but with the addition of $\mathrm{NaCl}$, the probability of formation of this phase is negligible. Thus the presence of $\mathrm{NaCl}$ does not allow particles to oxidize. The Rietveld analysis for sample $\mathrm{C}$ was carried out.

Figure 2 exhibits the Rietveld refined pattern. The parameters derived from the fit are given in table 1 . The particle diameter determined by Rietveld refinement $\left(D_{\text {ref }}\right)$ is in good agreement with those calculated using Scherrer's formalism.

The refined occupancy at tetrahedral A-site and octahedral B-site indicates that the sample suggests a mixed structure. The cation distribution obtained for our sample $\mathrm{C}$ agrees with those obtained by earlier workers for bulk cadmium ferrite (Yokoyama et al 1996). However, the positional parameter of oxygen is slightly higher than the expected value for ideal close packed arrangements

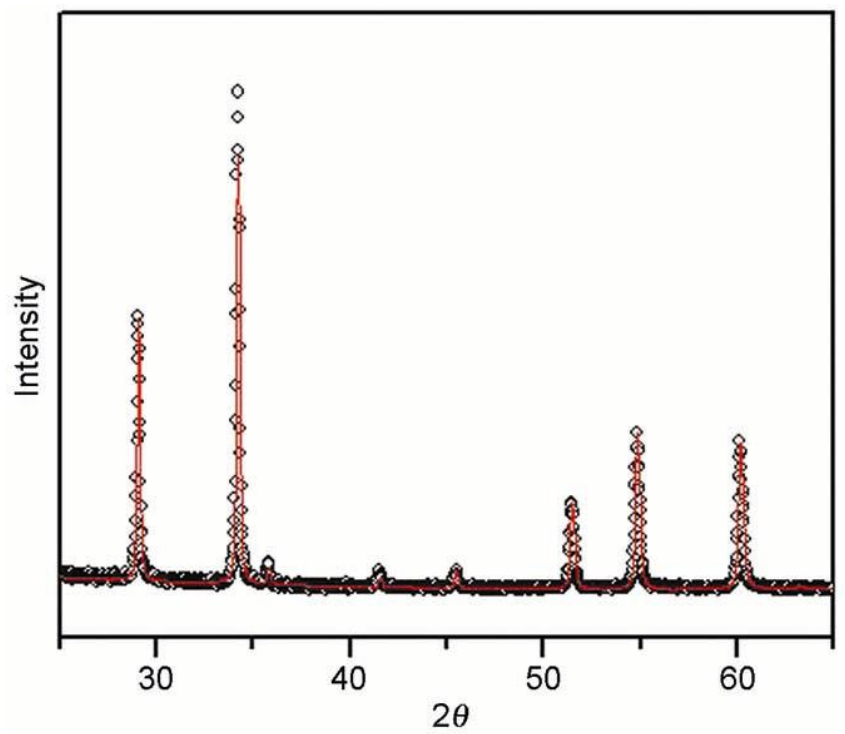

Figure 2. Rietveld refinement pattern for sample C. The Xray data are shown as dots; the solid line is best fit to the data. The lower curve represents the difference between the observed and calculated profiles.

Table 1. Structural parameters and Rietveld agreement for sample C, the description of the obtained parameters is done in the text.

\begin{tabular}{ll}
\hline Parameters & \multicolumn{1}{c}{ Sample } \\
\hline$x$ & $0 \cdot 03(5)$ \\
$u$ & $0 \cdot 256(2)$ \\
$a_{\text {(Ref) }}(\mathrm{nm})$ & $0 \cdot 8692(0 \cdot 3)$ \\
$a_{\text {(ASTM) }}(\mathrm{nm})$ & $0 \cdot 86972$ \\
$D_{\mathrm{Ref}}(\mathrm{nm})$ & 43 \\
$D_{\mathrm{XR}}(\mathrm{nm})$ & 45 \\
$\chi^{2}$ & $1 \cdot 11$ \\
$R_{\mathrm{P}}(\%)$ & $3 \cdot 57$ \\
$R_{\mathrm{wp}}(\%)$ & $4 \cdot 25$ \\
\hline
\end{tabular}


(equal to $0 \cdot 25$ ). The determined lattice constant from the XRD data agrees well with the ASTM tables (No. 221063). The above result indicates that the incorporation of $\mathrm{NaCl}$ not only inhibits growth of the particles but also helps to get better crystalline structure.

Morphology of the dried particles was measured using transmission electron microscopy. Figure 4 shows the TEM image of sample C. It is evident from figure 3 that most of the particles are nearly spherical. The inset shows the single particle image. Typical size ranges from 50$100 \mathrm{~nm}$.

To further confirm our argument of the formation of different phases we have carried out the non-destructive method of characterization such as infra-red spectroscopy. The IR absorption spectra recorded at room temperature in the range $1000-400 \mathrm{~cm}^{-1}$ is shown in figure 4 . It

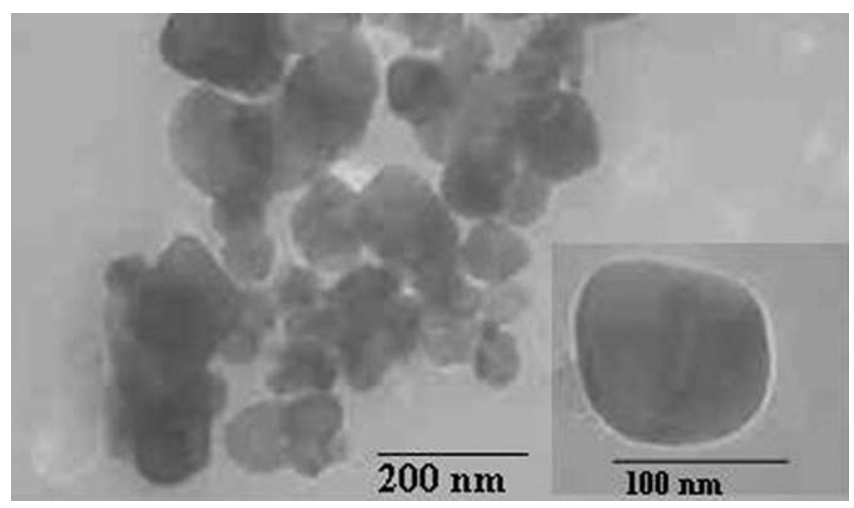

Figure 3. TEM image of sample C (Inset: single particle image).

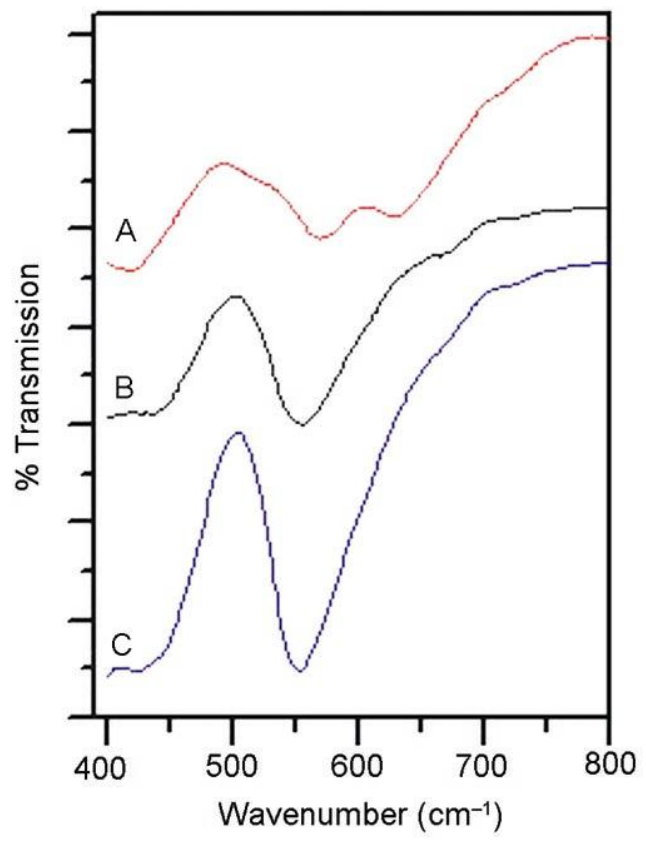

Figure 4. Infrared spectra for samples $\mathrm{A}, \mathrm{B}$ and $\mathrm{C}$ recorded at room temperature in $\mathrm{KBr}$ matrix. exhibits two main absorption bands at wave numbers $600 \mathrm{~cm}^{-1}\left(v_{1}\right)$ and $400 \mathrm{~cm}^{-1}\left(v_{2}\right)$. These are attributed to tetrahedral A and octahedral B sites of the spinel structure, respectively (Modi 2004). Sample A shows broad peaks around $416 \mathrm{~cm}^{-1}$ and $568 \mathrm{~cm}^{-1}$ indicating the formation of ferrite phase while peak at $628 \mathrm{~cm}^{-1}$ shows presence of the other phase. This agrees with the X-ray results. Samples $\mathrm{B}$ and $\mathrm{C}$ show bands assigned to ferrite phases only. The only difference in spectra is the sharpness of the peaks. The intense peaks at 420 and $560 \mathrm{~cm}^{-1}$ for sample $\mathrm{C}$ compared to $\mathrm{B}$ suggest the strong interaction at $\mathrm{B}$ and $\mathrm{A}$ sites, respectively for sample $\mathrm{C}$. Thus, sample $\mathrm{C}$ has more precise configuration of atoms or ions in the structure. These results are in agreement with our X-ray results.

For the nano-size spinel ferrite particle elastic modulus, Debye temperature etc parameters were calculated using the method given elsewhere (Modi 2004; Modi et al 2005) and the same are given in tables 1 and 2. Table 1 indicates that the average force constant, $k_{\text {avg }}$ value, obtained for sample $\mathrm{C}$ is comparable to the bulk $\mathrm{CdFe}_{2} \mathrm{O}_{4}$ (assuming that $\mathrm{Cd}^{+2}$ occupied A-site only). The slightly higher value obtained for Young's modulus $(E)$, bulk modulus $(B)$ and rigidity modulus $(G)$ indicates that the deformation of solid is difficult and that the solid has a strong tendency to 'spring' back to its equilibrium position (table 3 ). Theoretically, Poisson's ratio should remain in the range of 1$0 \cdot 5$. The obtained value of $\sigma$ is in conformity with the theory of isotropic elasticity. X-ray results show that particle size of sample B is slightly higher than sample C. It has been reported that the particle size and elastic moduli have an inverse relationship (Modi et al 2005). Based on this, observed higher values of sample B may be explained. The above IR result supports the conclusion that sample $\mathrm{C}$ is a well crystalline nano-magnetic particle of Cd-ferrite. Thus, in the following sections, we will concentrate on the magnetic properties of sample $\mathrm{C}$, only.

Figure 5 shows the magnetization curves for sample $\mathrm{C}$ recorded at $293 \mathrm{~K}$ and $5 \mathrm{~K}$. At both temperatures the magnetization curve do not saturate up to $10 \mathrm{kOe}$ field. This non-saturating behaviour indicates the presence of two components: (i) ferro/ferrimagnetic and (ii) super-paramagnetic or paramagnetic. The inset in figure 5 shows the ferro/ferrimagnetic contribution at room temperature after deducting the linear term contribution at high field. The saturation magnetization value thus obtained is $1.4 \mathrm{emu} / \mathrm{g}$. Finite coercivity values of $40 \mathrm{Oe}$ and $470 \mathrm{Oe}$ were also observed at $293 \mathrm{~K}$ and $5 \mathrm{~K}$, respectively. According to Kamiyama et al (1992), the small deviation from cation distribution of $\mathrm{Fe}^{3+}$ ions from B-site to A-site, results into localized magnetic clusters. The size and shape of these magnetic clusters are influenced by the strong A-B interaction between $\mathrm{Fe}$ ions on the A-sites and $\mathrm{Fe}$ ions on $\mathrm{B}$ sites. A random arrangement of Fe ions on both sites will yield many clusters with different volumes and magnetic order. Since the percentage of magnetic ions in the present case at the tetrahedral A-site $(0.03)$ is well below the 
Table 2. Lattice constant $(a)$, average crystallite size $(D)$, X-ray density $(\rho)$, band position $(v)$ and force constant $(k)$ for the samples $\mathrm{B}$ and $\mathrm{C}$ and bulk $\mathrm{CdFe}_{2} \mathrm{O}_{4}$.

\begin{tabular}{lccccccc}
\hline Sample code & $a(\mathrm{~nm})$ & $\begin{array}{c}\rho \times 10^{3} \\
\left(\mathrm{~kg} / \mathrm{m}^{3}\right)\end{array}$ & $\begin{array}{c}v_{1} \times 10^{3} \\
\left(\mathrm{~m}^{-1}\right)\end{array}$ & $\begin{array}{c}v_{2} \times 10^{3} \\
\left(\mathrm{~m}^{-1}\right)\end{array}$ & $\begin{array}{c}k_{\mathrm{t}} \times 10^{2} \\
(\mathrm{~N} / \mathrm{m})\end{array}$ & $\begin{array}{c}k_{0} \times 10^{2} \\
(\mathrm{~N} / \mathrm{m})\end{array}$ & $\begin{array}{c}\text { Avg. } k \times 10^{2} \\
(\mathrm{~N} / \mathrm{m})\end{array}$ \\
\hline $\mathrm{B}$ (without $\mathrm{NaCl})$ & 0.8695 & 5.819 & 553.47 & 435.83 & 2.62 & 1.12 & 1.87 \\
$\mathrm{C}$ (with $\mathrm{NaCl})$ & 0.8693 & 5.823 & 553.47 & 424.26 & 2.62 & 1.06 & 1.84 \\
$\mathrm{Bulk} \mathrm{CdFe}_{2} \mathrm{O}_{4}$ & 0.8700 & 5.81 & 554 & 412 & 2.57 & 1.00 & 1.79 \\
\hline
\end{tabular}

Table 3. Elastic wave velocity $(V)$, Young modulus $(E)$, bulk modulus $(B)$, rigidity modulus $(G)$, Poisson's ratio $(\sigma)$ and debye temperature $(\theta)$ for the samples $\mathrm{B}$ and $\mathrm{C}$ and bulk $\mathrm{CdFe}_{2} \mathrm{O}_{4}$.

\begin{tabular}{|c|c|c|c|c|c|c|c|c|}
\hline Sample code & $V_{1}(\mathrm{~m} / \mathrm{s})$ & $V_{\mathrm{s}}(\mathrm{m} / \mathrm{s})$ & $V_{\mathrm{m}}(\mathrm{m} / \mathrm{s})$ & $E(\mathrm{GPa})$ & $B(\mathrm{GPa})$ & $G(\mathrm{GPa})$ & $\sigma$ & $\theta(\mathrm{K})$ \\
\hline B & $6079 \cdot 32$ & $3509 \cdot 89$ & $3896 \cdot 62$ & 193.53 & $215 \cdot 06$ & 71.68 & $0 \cdot 35$ & $510 \cdot 31$ \\
\hline $\mathrm{C}$ & $6029 \cdot 00$ & $3480 \cdot 84$ & $3864 \cdot 36$ & $190 \cdot 48$ & $211 \cdot 66$ & $70 \cdot 55$ & $0 \cdot 35$ & $506 \cdot 21$ \\
\hline Bulk CdFe ${ }_{2} \mathrm{O}_{4}$ & $5941 \cdot 50$ & $3430 \cdot 30$ & $3810 \cdot 90$ & $185 \cdot 00$ & $206 \cdot 00$ & $68 \cdot 00$ & $0 \cdot 35$ & 499 \\
\hline
\end{tabular}
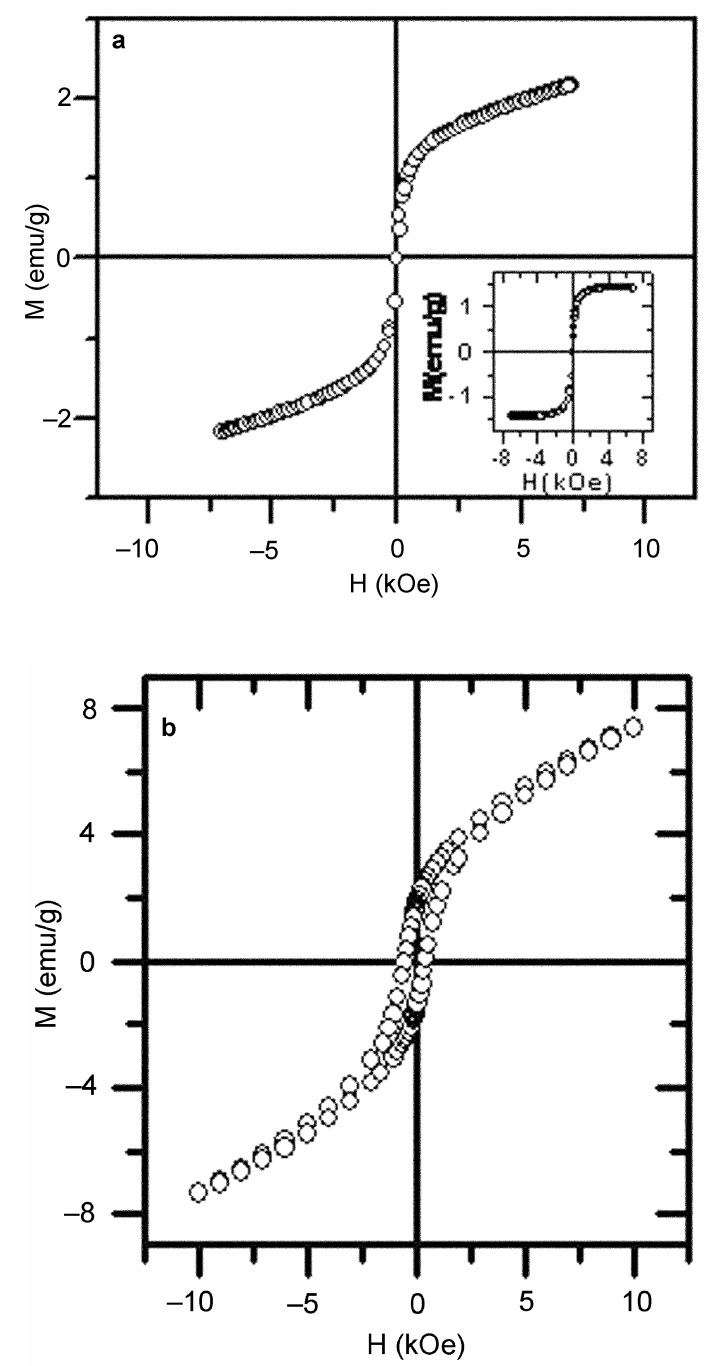

Figure 5. Magnetic hysteretic loops at a. room temperature $293 \mathrm{~K}$, and b. $5 \mathrm{~K}$ for sample C. (Inset: room temperature magnetization curve after subtracting the linear contribution. See text). percolation threshold for A-site $(0.33)$ (Hubsch et al 1978), a long range ferro-ferrimagnetic ordering cannot exist. Recently, O'Silva and Morais (2005) investigated canting in $\mathrm{CdFe}_{2} \mathrm{O}_{4}$ magnetic fluid using magnetic resonance technique. Using angular dependence of resonance field they have shown that in fine particle of cadmium ferrite, a different spin structure exists, viz. a core-shell structure. In this core-shell model, core is anti-ferromagnetic while the shell structure is characterized by a canting like spin structure. The anti-ferromagnetic core volume fraction will increase with the increase in size, which results into decrease in magnetization. Therefore, in the present case the observed non-saturating behaviour at 293 and $5 \mathrm{~K}$ indicates magnetic clusters having surface dominated anisotropy (particle size, $<60 \mathrm{~nm}$ ). Under this case one can consider the particle having a large anti-ferromagnetic core with canted surface spins. The anisotropy of the surface spin can give rise to coercivity observed at $293 \mathrm{~K}$ and $5 \mathrm{~K}$. The increase in coercivity at $5 \mathrm{~K}$ indicates that the surface spins dominate at low temperature. The temperature dependent magnetization at $10 \mathrm{kOe}$ field (figure 6a) shows a maximum at $10 \mathrm{~K}$ and change in curvature at around $100 \mathrm{~K}$. The observed maximum at $10 \mathrm{~K}$ is attributed to antiferromagnetic ordering arising from the core, this value agrees with that reported for the bulk cadmium ferrite. The observed change in curvature at $100 \mathrm{~K}$ indicates the blocking temperature of magnetic clusters having canted surface spins. This effect is much evident in the zero field cooled (ZFC) and field cooled (FC) magnetization curves (figure $6 \mathrm{~b}$ ). Under $\mathrm{ZFC}$ and $\mathrm{FC}$ conditions, the magnetization showed a down trend at $\sim 20 \mathrm{~K}$ irrespective of the magnitude of magnetic field (5-1000 Oe). Thus confirming that core is anti-ferromagnetic having an ordering temperature of $<15 \mathrm{~K}$. Due to two competing interactions between anti-ferromagnetic core and canted surface spins, the peak at $10 \mathrm{~K}$ in $M$ vs $T$ curve is broader in $\mathrm{ZFC}$ for low field cooled values. But at $1 \mathrm{kOe}$ the $\mathrm{ZFC}$ peaks around $15 \mathrm{~K}$. 
In addition, the $\mathrm{ZFC}$ and $\mathrm{FC}$ curves show a hump at around $100 \mathrm{~K}$ (in low field cooled condition it is more prominent, figure $6 \mathrm{~b}$ ) and irreversibility starts for 5 Oe field right from $300 \mathrm{~K}$. On the other hand, for sample cooled in $1 \mathrm{kOe}$ field this irreversibility starts from $100 \mathrm{~K}$. The broad hump at $100 \mathrm{~K}$ and irreversibility are found to be independent of the cooling field magnitude. This contradicts the ferro and/or ferrimagnetic type of ordering in the system in which the blocking temperature shifts with field cooling conditions. These observed results can be explained as follows. (i) For low field cooled condition, the applied field is much less than the coercive field at room temperature $(40 \mathrm{Oe})$. Therefore, irreversibility starts from $293 \mathrm{~K}$, (ii) at $100 \mathrm{Oe}$ and $1 \mathrm{kOe}$, the applied magnetic field is greater than coercive field at room temperature; therefore,
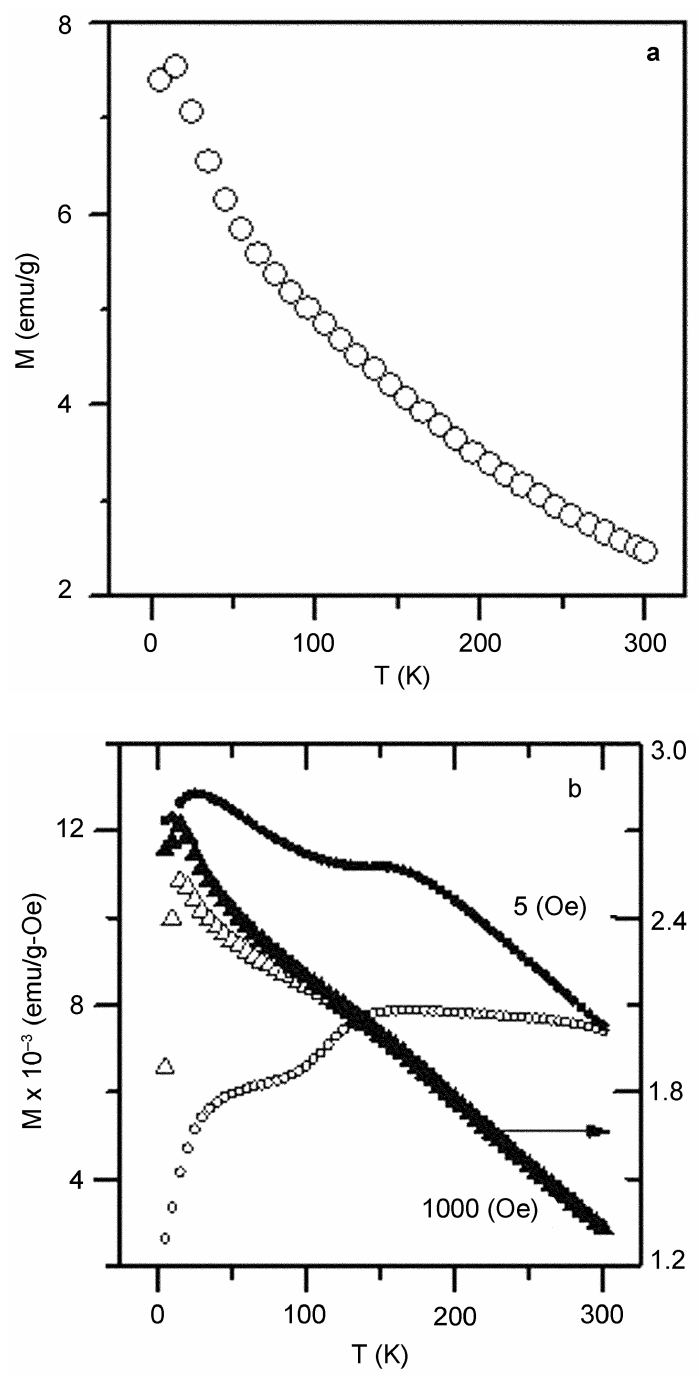

Figure 6. a. Temperature dependent magnetization measured at $H=10 \mathrm{kOe}$ for sample $\mathrm{C}$ and $\mathbf{b}$. temperature dependence of magnetization for sample $\mathrm{C}$ measured at $H=5 \mathrm{Oe}$, and $1 \mathrm{kOe}$ under zero field (ZFC, open symbol) and field cooled (FC, filled symbol) conditions. one observes the merging of FC and ZFC curves, (iii) the observed field independent behaviour of the irreversibility of FC and ZFC indicates that the surface anisotropy due to canted surface spins dominates below $100 \mathrm{~K}$ and (iv) this surface anisotropy also contributes to the hysteresis observed at $293 \mathrm{~K}$ and $5 \mathrm{~K}$ as suggested by O'Silva and Morais (2005).

Figures 7a,b show the frequency dependent real and imaginary parts of the susceptibilities for sample $\mathrm{C}$ under 1 Oe a.c. fields. The real part of the a.c. susceptibility (figure 7a) shows a broad maximum at around $125 \mathrm{~K}$. This is also reflected in the imaginary part of the a.c. susceptibility (figure 7b). These observed peaks are inde-
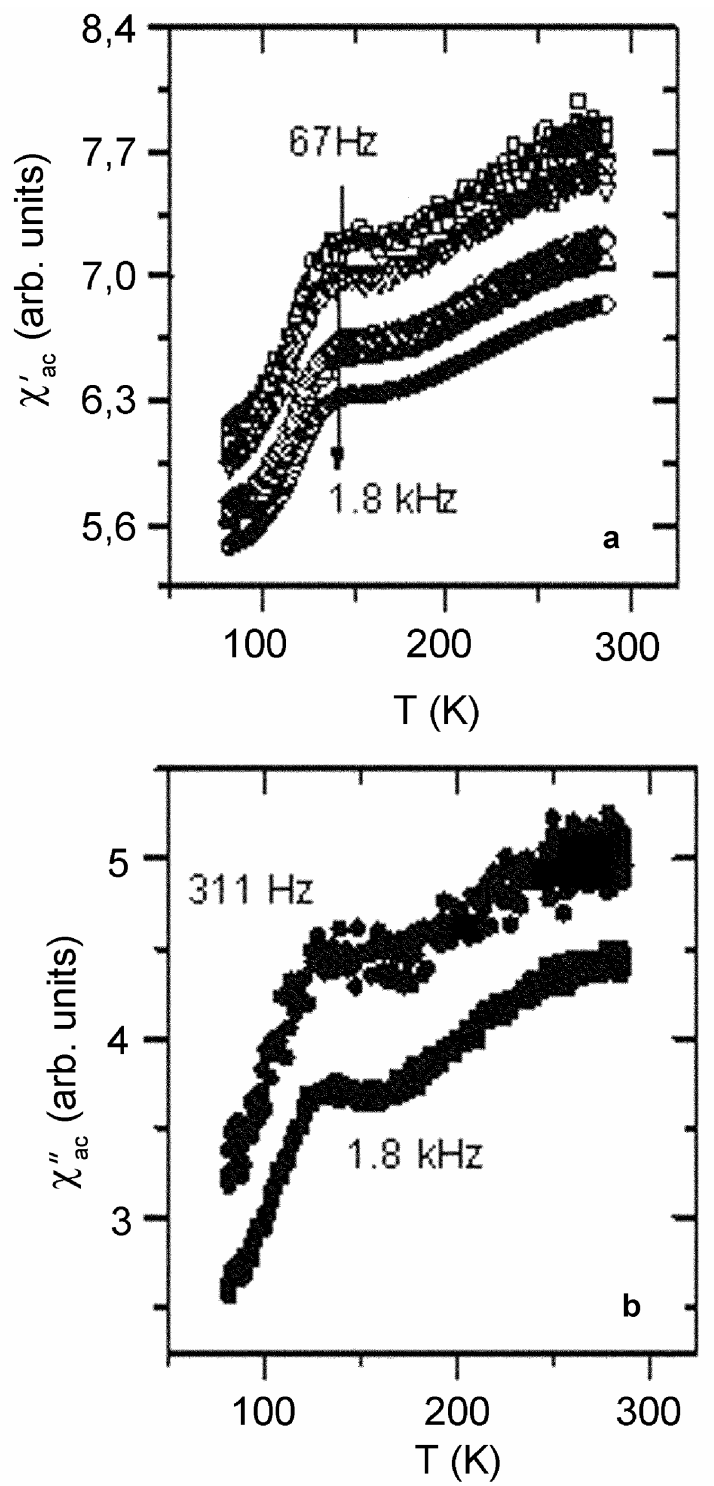

Figure 7. Temperature and frequency dependence a.c. susceptibility measured with an a.c. field of 1 Oe for sample $\mathrm{C}$, a. real part $\left(\chi_{\mathrm{ac}}^{\prime}\right)$ and $\mathbf{b}$. imaginary part $\left(\chi_{\mathrm{ac}}^{\prime \prime}\right)$. (For imaginary part, only data of two frequencies are shown for clarity). 
pendent of frequency, suggesting that the observed magnetic ordering is not a volume effect i.e. the spin disorder is not in entire volume of the particles, but it is only at the surface. These observations are consistent with the magnetic resonance study on cadmium ferrite magnetic fluid by O’Silva and Morais (2005).

\section{Conclusions}

The present investigations suggest that it is possible to synthesize the cadmium ferrite using this new co-precipitation synthesis route using $\mathrm{NaCl}$ as controlling catalyst at low annealing temperature and significantly reduced time interval. The bulk magnetic properties are still retained even though the particle size is below $60 \mathrm{~nm}$. The observed magnetic properties can be explained on the basis of an anti-ferromagnetic core with a shell containing 'ferromagnetic-like', but canted spin structure.

\section{Acknowledgements}

One of the authors (RVU) is thankful to the Gujarat Council of Science and Technology (GUJCOST), Gandhinagar, for the project funding and the Swedish Agency STINT for a visiting fellowship. Part of the work was carried out under the Department of Science and Technology project No. DST-M-15 and the Council of Scientific and Industrial Research project No. ESS/21(0537)/ 02/EMR-II.

\section{References}

Chinnasamy C N et al $2001 \mathrm{~J}$. Appl. Phys. 90527

Elkins K, Li D, Poudyal N, Nandwana V, Jin Z, Chen K and Liu J Ping 2005 J. Phys. D: Appl. Phys. 382306

Hastings J M and Corliss L M 1956 Phys. Rev. 1021460

Herzer G, Vaznez M, Knobel M, Zhukov A, Reininger T, Davies H A, Grossinger R and Sanchez Li J L 2005 J. Magn. Magn. Mater. 294252

Hubsch J, Gavoille G and Bolfa J 1978 J. Appl. Phys. 491363

Kamiyama T, Haneda K, Sato T, Ikeda S and Asano H 1992 Solid State Commun. 81563

Lotgering F K 1966 J. Phys. Chem. Solids 27139

Mahmoud M H, Abdallas A M, Hamdeh H H, Hikal W M, Taher S M and Ho J C 2003 J. Magn. Magn. Mater. 263269

Modi K B 2004 J. Mater. Sci. 392887

Modi K B, Sharma P U, Chhatbar M C and Joshi H H $2005 \mathrm{~J}$. Mater. Sci. 401247

Sato T, Haneda K, Seki M and Iijima T 1987 Proceedings of the international symposium on the physics of magnetic materials (Singapore: World Scientific) p. 210

Sato T, Haneda K, Seki M and Iijima T 1990 Appl. Phys. A50 13

Sato T, Haneda K, Iijima T and Seki M 1992 Proc. 6th int. conf. ferrites (Tokyo and Kyoto: Japan Society of Powder and Powder Metallurgy) p. 984

Silva O and Morais P C 2005 J. Magn. Magn. Mater. 289136

Ström Valter 1998 Integral and local a.c. susceptibilityinstruments and applications, Ph D Thesis, Royal Institute of Technology, Stockholm, Sweden

Yokoyama M, Sato T, Ohta E and Sato T 1996 J. Appl. Phys. 801015

Yokoyama M, Ohta E, Sato T and Sato T 1998 J. Magn. Magn. Mater. 183173 